\title{
Role of CT Portography in Detection of Portosystemic Collaterals in Patients with Portal Hypertension
}

\author{
Mohamed E. Abo El-Maaty, Haitham M. Nasr, Wagdy N. Anwar \\ Department of Radiology \\ Faculty of Medicine- Ain Shams University
}

\begin{abstract}
Background: CT and CT portography become the tools of investigation of the liver and the portosystemic varices by drawing their course to avoid bleeding which could be life-threatening, and in major operations such as liver transplantation.

Aim of the Work: to draw portosystemic collaterals associated with portal hypertension by CT Portography imaging.

Patients and Methods: In this study, we have assessed 20 cases in 6 months at the Ain Shams university hospitals, where they subjected to full history sheeting, full clinical examination, MDCT portography and to the upper GI endoscopy.

Results: There is significant correlation between portal vein diameter and sites of collaterals, as the sites of collaterals increase when the diameter of the portal vein decreases. Regarding the CT findings and their comparison with the upper GI endoscopy findings was found that all the cases that were positive for gastroesophageal collaterals in CT were also positive for gastro-esophageal collaterals in the upper GI endoscopy, which is meaning that the CT is very good excluded modality with $100 \%$ sensitivity. While the CT was upgrading for 2 cases from 20 cases in gastric varices (10\%), 3 cases from 20 cases(15\%) in esophageal varices, which is meaning that $\mathrm{CT}$ has $89 \%, 82 \%$ specificity in grading the gastric and esophageal collaterals respectively.

Conclusion: MDCT portography is an important imaging modality in the assessment of the portal vein, the resulting portosystemic collaterals. It is very good screening modality for any suspected varices as it has 100 $\%$ sensitivity.
\end{abstract}

Keywords: CT Portography, portosystemic collaterals, portal hypertension

\section{Introduction}

In portal hypertension, the hepatoportal blood flow is directed away from the liver through collateral pathways to low pressure systemic vessels. Among them, there are the esophageal varices, which are the most important clinically because they are frequent source of gastrointestinal bleeding. Information about other collateral pathways is considered relevant, especially if interventional procedures or surgery is contemplated because the probable disruption of these veins can cause significant bleeding ${ }^{(\mathbf{1})}$.

Hepatic venous pressure gradient (HVPG) is currently considered as the "gold standard although, it is restricted technique because it is difficult and invasive while direct measurement of portal pressure (PP) is rarely performed due to significant invasiveness and potential complications (2).

Diagnostic angiographic procedures include both direct and indirect portography, indirect portography involves arterial injections into the superior mesenteric artery (SMA) or splenic artery and requires a prolonged arterial infusion of contrast material (CM) followed by delayed imaging. These techniques are also invasive and limited by flow dynamics ${ }^{(3)}$.

Patients with cirrhosis should undergo screening for esophageal varices using upper gastrointestinal endoscopy because the bleeding from these varices is very lifethreatening ${ }^{(4)}$.

Up to $30 \%$ of patients screened by endoscopy found to have moderate to large varices (> or $=5 \mathrm{~mm}$ diameter) which are at high risk of bleeding ${ }^{(5)}$.

Many years ago angiography was considered the standard for the detection of varices. but with the possibilities and availability of crosssectional imaging techniques, collateral vessels could be now well demonstrated in all parts of the abdomen and thorax without any risk of invasiveness ${ }^{(\mathbf{1})}$.

Noninvasive techniques include the color Doppler ultrasound (US), which depicts the portal vein and provides good information 
about the velocity and the flow direction, but it is operator dependent, while in patients with potential portosystemic shunt you need a procedure, that covers the whole portal venous system ${ }^{(6)}$.

CT and CT angiography has become powerful procedures for examination of the liver. The addition of a portal-phase acquisition with 3D vascular reconstruction can augment the surgeon's perception of potentially problematic varices by detailing the course of these tortuous vessels. This is extremely important for many surgeries to avoid any possible disaster ${ }^{(7)}$.

\section{Aim of the Work}

The purpose of our study is to deled portosystemic collaterals associated with portal hypertension by CT Portography imaging.

\section{Patients and Methods}

Our study includes assessment of 20 cases in 6 months, patients are referred to the internal medicine department at the Ain Shams university hospital, where they examined, thin referred to the radiology department, where they investigated.

The study included patients with evidence of portal hypertension (clinical and previous imaging) and evidence of liver cirrhosis.

- Patients with impaired renal function, pregnant patients, contrast hypersensitivity, vitally unstable and hepatic encephalopathy were excluded from the study.

The patients were subjected to the following: Full history sheet, full clinical and laboratory examination, CT portography and upper GI endoscopy, the history sheet included name, age, sex, history of viral hepatitis, past history of hematemesis or melena. The laboratory examination includes Liver enzymes (SGOT, SGPT), hepatitis markers (HBsAg, HCV Abs), renal function tests (creatinine level).

\section{The technique of our CT examination}

\section{CT machine that is used:}

- Toshiba -Aquillion, helical CT scanner 64 detectors in Ain shams university hospital. Patients were instructed to food fasting for 6 hours before the examination to avoid vomiting when the $\mathrm{CM}$ injected. Revision of all patients' personal and laboratory data was conducted to be sure that they are within normal range. Patients were informed about the CT scan and how to hold breath during the examination when it requested. Patients lied in the supine position on the CT table with head first and arms in resting position.

- A wide bore cannula is placed in the antecubital vein and to ensure the good function of the cannula $10 \mathrm{~mm}$ saline is injected first. The scout is determined in the coronal view and the scan is planed from the bifurcation of trachea to the symphysis pubis pre and post-contrast. The scan then proceeds with beam collimation $4 \times 1 \mathrm{~mm}$; table feed 5 $\mathrm{mm}$ per rotation (pitch 1.25); and rotation time $0.5 \mathrm{sec}$. Image reconstruction was performed with a slice thickness of $1.25 \mathrm{~mm}$ at an interval of $0.6 \mathrm{~mm}$. with $120 \mathrm{kV}, 240-280 \mathrm{~mA}$. 100 $120 \mathrm{ml}$ of the $\mathrm{CM}$ is injected at a rate of $4-5$ $\mathrm{ml} / \mathrm{sec}$ by a power injector and the scan proceeded in the following stations. Arterial phase: at $18-20 \mathrm{sec}$ from injection, helical scanning is started from tracheal bifurcation to the symphysis pubis in craniocaudal direction with (1.25 collimation, 0.6 pitch, $120 \mathrm{kV}, 240$ $-280 \mathrm{~mA})$. Portal phase: $60 \mathrm{sec}$ from injection ( 2.5 collimation, 0.6 pitch, $120 \mathrm{kV}, 240-280$ $\mathrm{mA}$ ), but the scan is done in the reverse direction. Delayed phase: at $200 \mathrm{sec}$ from injection through the entire liver with $(2.5$ collimation, 0.6 pitch, $120 \mathrm{kV}, 240-280 \mathrm{~mA}$ ).

\section{Image processing:}

All data were acquired and then analyzed with a standard algorithm, post-processed and reconstructed at the workstation that allows the generation of 3D images. The MIP was used for the $3 \mathrm{D}$ reconstruction images that are good in detection of the details of the veins. The images were reconstructed with 1.5 collimation. 7 position increments.

\section{Image interpretation:}

All images were analyzed for Portal vein diameter and patency, CT evidence for portal hypertension as ascites or splenomegaly, complete analysis of the collaterals that were present, detection of all types of these collaterals, any hepatic focal lesions, and HCC.

\section{Image display:}

All images were sent to a work station that allows interactive analysis and also was copied on hard copies. 
SOHAG MEDICAL JOURNAL

Vol. 23 No. 3 July 2019

\section{Statistical analysis:}

Data were coded and entered using the statistical package SPSS (Statistical Package for the Social Sciences) version 24. Data were summarized using mean, standard deviation, median, minimum and maximum in quantitative data and using frequency (count) and relative frequency (percentage) for categorical data. Comparisons between quantitative variables were done using the non-parametric Mann-Whitney test. For comparing categorical data, Chi-square $\left(\chi^{2}\right)$ test was performed. The exact test was used instead when the expected frequency is less than 5. Standard diagnostic indices including sensitivity, specificity, positive predictive value (PPV) and negative predictive value (NPV) were calculated. A P-value of less than 0.05 was considered as statistically significant.

\section{Results}

\begin{tabular}{||c|c|c|c||}
\hline \multicolumn{2}{|c|}{} & Count (20 Pts) & \% \\
\hline PV dilatation & Yes & $\mathbf{9}$ & $\mathbf{4 5 . 0 \%}$ \\
& No & $\mathbf{1 1}$ & $\mathbf{5 5 . 0 \%}$ \\
\cline { 2 - 4 } & & & $\mathbf{2 5 . 0 \%}$ \\
\hline PV thrombosis & Yes & $\mathbf{5}$ & $\mathbf{7 5 . 0 \%}$ \\
\hline
\end{tabular}

Table (1): Descriptive table showing the distribution of the studied patients regarding their PV patency and diameter.

\begin{tabular}{|c|c|c|c|c|c|c|c|c|c|c|c|}
\hline & \multicolumn{10}{|c|}{ PV dilatation } & \multirow{3}{*}{ P-value } \\
\hline & \multicolumn{5}{|c|}{ Yes } & \multicolumn{5}{|c|}{ No } & \\
\hline & $\sum^{\tilde{E}}$ & \% & 恶 & $\stackrel{\Xi}{\Xi}$ & 志 & $\sum_{\Sigma}^{\Xi}$ & \% & 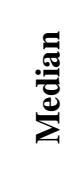 & $\underset{\Xi}{\Xi}$ & 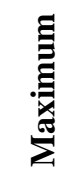 & \\
\hline Sites of collateral by CT & 4.00 & .87 & 4.00 & 3.00 & 5.00 & 6.00 & 1.05 & 6.00 & 4.00 & 8.00 & .0009 \\
\hline
\end{tabular}

Table (2): Correlation between PV diameter and sites of portosystemic collaterals in the studied patients.

There is a significant correlation between the PV diameter \& sites of collaterals as $\mathrm{P}<\quad 0.001$. The increase in collateral sites is associated with decrease in PV diameter.

\begin{tabular}{||c|c|c||}
\hline \multirow{2}{*}{ Findings } & \multicolumn{2}{|c|}{ Patients (20) } \\
\cline { 2 - 3 } & Number & \multicolumn{2}{c|}{} \\
\hline Cirrhosis & 17 & $10 \%$ \\
\hline HFL & 2 & $5 \%$ \\
\hline HCC & 1 & $70 \%$ \\
\hline Splenomegaly & 14 & $90 \%$ \\
\hline Ascites & 18 & $25 \%$ \\
\hline PV thrombosis & 5 & \\
\hline
\end{tabular}

Table (3): $\quad$ Distribution of all studied patients regarding other portal hypertension radiological criteria and common associations. 
SOHAG MEDICAL JOURNAL

Vol. 23 No. 3 July 2019

\begin{tabular}{|c|c|c|}
\hline \multirow{2}{*}{ Type of detected collaterals } & \multicolumn{2}{|c|}{$\begin{array}{c}\text { Frequency } \\
(\mathrm{n}=20 \text { Patients) }\end{array}$} \\
\cline { 2 - 3 } & Number & \% \\
\cline { 2 - 3 } & 14 & $70 \%$ \\
\hline Perisplenic & 11 & $55 \%$ \\
\hline Periumbilical & 9 & $45 \%$ \\
\hline Splenorenal & 8 & $40 \%$ \\
\hline Esophageal & 8 & $40 \%$ \\
\hline Anterior abdominal wall & 7 & $35 \%$ \\
\hline Perigastric & 5 & $25 \%$ \\
\hline Portahepatic & 1 & $5 \%$ \\
\hline Rectal & \multicolumn{2}{|c|}{} \\
\hline
\end{tabular}

Table (4) Distribution of the studied patients regarding frequency of the detected portosystemic collaterals.

\begin{tabular}{|l|l|l||}
\hline & Portal vein dilatation & Portal vein thrombosis \\
\hline Percentage of cases of collaterals & $\mathbf{8 0} \%$ & $20 \%$ \\
\hline Frequent site of collaterals & Perisplenic & Portahepatic \\
\hline
\end{tabular}

Table (5): Distribution of the collaterals regarding the portal vein patency.

According to the above table, 4 cases from the 5 cases of portal vein thrombosis had also portosystemic collaterals with a percentage $80 \%$ and the main site for these collaterals was the porta hepatis.

\begin{tabular}{||c|c|c||}
\hline & Count (20Pts) & \% \\
\hline I & 13 & $65 \%$ \\
\hline II & 1 & $5 \%$ \\
\hline III & 3 & $15 \%$ \\
\hline IV & 3 & $15 \%$ \\
\hline
\end{tabular}

Table (6): Grading of the studied patients who have esophageal varices by CT. Table (6): Grading of the studied patients who have esophageal varices by CT.

\begin{tabular}{|c|c|c|}
\hline & Count (20Pts) & \% \\
\hline I (Negative for varices) & 13 & $65 \%$ \\
\hline II & 4 & $20 \%$ \\
\hline III & 1 & $5 \%$ \\
\hline IV & 2 & $10 \%$ \\
\hline
\end{tabular}

Table (7): Grading of the studied patients who have esophageal varices by endoscopy.

\begin{tabular}{|c|c|c|}
\hline & Count (20Pts) & $\%$ \\
\hline I & 15 & $75 \%$ \\
\hline II & 1 & $5 \%$ \\
\hline III & 3 & $15 \%$ \\
\hline IV & 1 & $5 \%$ \\
\hline
\end{tabular}

Table (8): Grading of the studied patients who have gastric varices by CT.

\begin{tabular}{|c|c|c|}
\hline \hline & Count (20Pts) & \% \\
\hline I (Negative for varices) & 15 & $75 \%$ \\
\hline II & 3 & $15 \%$ \\
\hline III & 1 & $5 \%$ \\
\hline IV & 1 & $5 \%$ \\
\hline
\end{tabular}

Table (9): Grading of the studied patients who have gastric varices by endoscopy. 
The detected varices by CT could be graded according to Yu et al. (8) as:

with a 4-point confidence scale for the development of variceal bleeding according to their maximum diameter (scores 1-4): (1) definitely low or no -risk or no varices; (2) probably low-risk (1-2 $\mathrm{mm}$ in maximum diameter); (3) probably high-risk(2-3mm in maximum diameter); and (4) definitely high-risk(more than $3 \mathrm{~mm}$ in maximum diameter).

\begin{tabular}{|c|c|}
\hline Statistic & Value \\
\hline Sensitivity & $100.00 \%$ \\
\hline Specificity & $82 \%$ \\
\hline
\end{tabular}

Table (10): CT sensitivity \& specificity in esophageal varices compared to endoscopy.

\begin{tabular}{||c|c|}
\hline Statistic & Value \\
\hline Sensitivity & $100.00 \%$ \\
\hline Specificity & $89 \%$ \\
\hline
\end{tabular}

Table (11) : CT sensitivity \& specificity in gastric varices compared to endoscopy

\section{According to the mentioned tables:}

(1) CT portography has $100 \%$ sensitivity and $82 \%$ specificity in detection of the "high risk" esophageal varices compared to endoscopy (Tables 6,7,10).

(2) CT portography has $100 \%$ sensitivity and $89 \%$ specificity in detection of the "high risk" gastric varices compared to endoscopy.(Table 8,9,11).

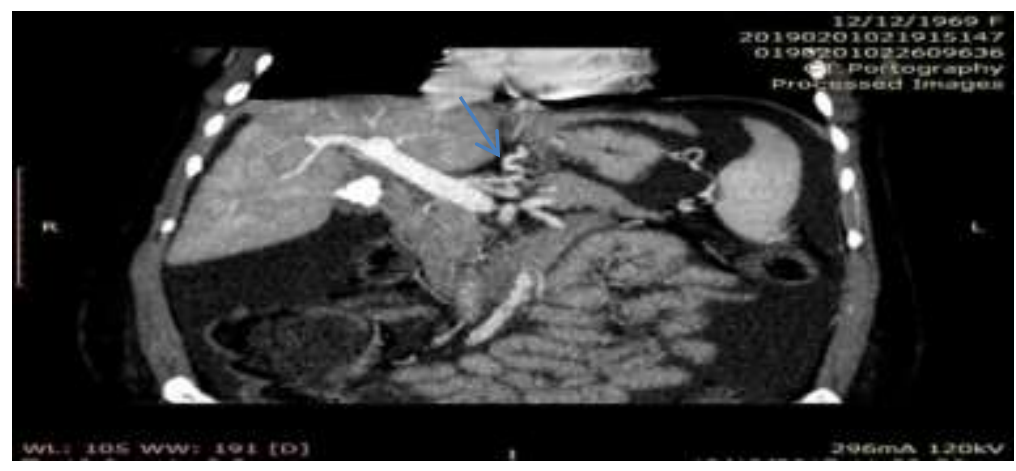

(A)

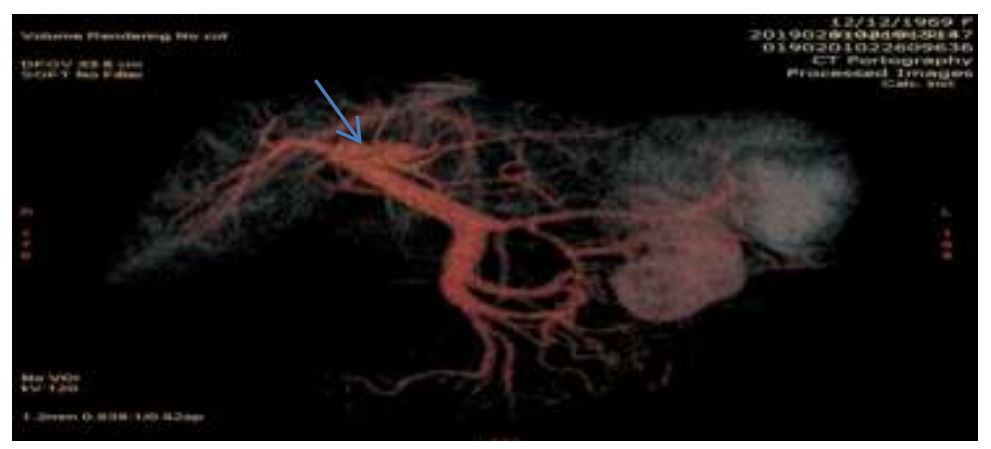

(B)

Female patient 50 years old with a history of chronic liver disease complains of marked abdominal distension and lower limb edema.

The endoscopic findings: enlarged tortuous esophageal varices grade II

The MDCT findings: cirrhotic liver with ascites, dilated portal vein and porta hepatic collaterals grade III. (A) Triphasic CT (portal) coronal reconstruction image showing dilated porta hepatic collaterals (the blue arrow). (B) CT with 3D reconstruction showing dilated porta hepatic collaterals (the blue arrow ) 


\section{Discussion}

The portal system is the system that carries the blood from the digestive system to the liver, MDCT portal venography can depict the extent and location of the portosystemic collaterals in patients with portal hypertension (9)

In patients with cirrhotic liver, portal hypertension could develop, so many collaterals could arise as alternative pathways called portosystemic collaterals, for many years the angiography was the standard for their detection ${ }^{(\mathbf{1 0})}$.

MDCT portal venography can display the portosystemic collaterals, as it has become a thin slice and better spatial resolution ${ }^{(11)}$.MDCT is also useful in diagnosis of other findings associated with portal hypertension such as ascites, liver cirrhosis, hepatic focal lesions, other collateral sites that can not be examined by the GI endoscopy ${ }^{(\mathbf{1 1})}$.

According to this study was found that the CT portography is very good technique for detecting the size and type of the collaterals with $100 \%$ sensitivity with the upper GI endoscopy, but it shows less accuracy in grading of the high-risk varices compared to the upper GI endoscopy, as it shows $82 \%$ specificity in esophageal varices while $89 \%$ specificity in fundal or gastric varices.

Also in other studies like (El-Kammash et al, (12), it was also found that the MDCT with MIP abilities is a reliable, noninvasive technique and able to depict the esophageal and other portosystemic collaterals.

In this study was found that 18 cases of 20 cases $(90 \%)$ have hepatic causes of portal hypertension while 1 case due to periportal fibrosis and another due to Budd-Chiari syndrome. In agreement, the results of Agarwal et al, ${ }^{(9)}$ found that the most common cause of portal hypertension has hepatic etiology with a percentage of $75 \%$.

According to Kim et al. ${ }^{(13)}$ who reported that the MDCT is a reliable, noninvasive modality for both portosystemic and hepatocellular carcinoma (HCC). Our study has also revealed the HCC in about $5 \%$ of the total number of the studied cases.

In our study the CT portography was able to classify the portosystemic collaterals according to the anatomical site and their grading, this data could be of benefit for the major operations such as liver transplantation, which is in agreement with Henseler et al. ${ }^{(7)}$, who reported that the variceal orientation and relationship to the adjacent organs are deceiving in this operation.

In this study were the prespleenic collaterals the most common type among the portosystemic collaterals which is in agreement with Antunez et al. ${ }^{(14)}$.

During the examination of the twenty patients, who have portal hypertension in this study for detection the portosystemic collaterals, that could arise, has revealed that

The CT portography was able to detect early the esophageal varices that could arise as a complication to the high pressure in the portal vein with $100 \%$ sensitivity to the upper GI endoscopy.

- Also in the gastric varices was the CT portography able to early detection of these varices with the same sensitivity $100 \%$ to the upper GI endoscopy

This is meaning that the CT portography could be used as screening tool for early detection of the portosystemic collaterals and prevention of their life-threating complications, as the MDCT portography in comparison to the upper GI endoscopy less annoying and more comfortable than the upper GI endoscopy, in addition to it is reliable, available and noninvasive technique, this is in agreement with Hegab et al. ${ }^{(15)}$, who reported that the CT portography can replace or serve as alternative to the upper GI endoscopy in screening patients with portal hypertension.

In the grading of the detected portosystemic collaterals was the MDCT portography not in the same accuracy with the upper GI endoscopy, as it was upgraded for the esophageal varices in 3 cases from the 20 studied cases from the low-risk zone (grade II) to the high-risk zone (grad III, IV) with percentage of specificity reached up to $82 \%$

While in the gastric varices was the CT portography upgrading in 2 cases from the 20 studied cases from the low-risk zone (grade II) to the high-risk zone (grad III,IV). This revealed that the $\mathrm{CT}$ portography has specificity $89 \%$ compared to the upper GI endoscopy. This is in agreement with Kim et 
al. ${ }^{(\mathbf{1 3})}$ who reported that careful examination of these portosystemic collaterals could be possible to void performing the upper GI endoscopy.

\section{Conclusion \\ Our study has found that:}

- MDCT portography is an important imaging modality in assessment the portal vein, portal hypertension and the resulting portosystemic collaterals.

- The MDCT portography can provide all data necessary for major surgeries such as liver transplantation.

- CT portography is very good screening modality for any suspected varices as it has $100 \%$ sensitivity.

- CT portography is considered less accurate than the upper GI endoscopy in variceal grading.

- CT portpgraphy can detect the portal vein thrombosis.

\section{References}

1. Cho, K. C., Patel, Y. D., Wachsberg, R. H., \& Seeff, J. Varices in portal hypertension: evaluation with CT. Radiographics, 1995;15(3), 609-622.

2. Thabut D, Moreau R, Lebrec D. Noninvasive assessment of portal hypertension in patients with cirrhosis. Hepatology. 2011 Feb; 53(2):683-94

3. Sehgal M, and Haskal ZJ. Use of transjugular intrahepatic portosystemic shunts during lytic therapy of extensive portal splenic and mesenteric venous thrombosis long term follow up. Jvasc Interv Radiology ,2000;11:6165 .

4. Triantos, C., \& Kalafateli, M. Endoscopic treatment of esophageal varices in patients with liver cirrhosis. World Journal of Gastroenterology: WJG, 2014; 20(36), 13015.

5. Ripoll L, Ayuso P, Baldello M. management of varices using endoscope. Diagn Interv Radiol. , 2007; 19:230-250

6. Wachberg RH, Bahramipour P, Sofocleous CT. Heptofugal flow in portal venous system: Pathophysiology imaging and diagnostic bitfal. Radiografic , 2002;22:123-14

7. Henseler, K. P., Pozniak, M. A., Lee Jr, F. T., \& Winter, T. C. Three-dimensional CT Angiography of Spontaneous Portosystemic Shunts 1. Radiographics, 2001;21(3), 691-704.
8. Yu NC, Margolis D, Hsu M, Raman S, Lu D. Detection and grading of esophageal varices on liver CT: comparison of standard and thinsection multiplanar reconstructions in diagnosticaccuracy.AJR, 2011;197(3):643-8.

9. Agarwal A, and Jain M. Multi detector CT portal venography in evaluation of prtosystemic collaterals Radiology. J Med Imaging Radiation , Radiation oncology, 2008;10:1440

10. Kang HK, Feong YY, Choi FH. Threedimensional Multi-Detector Row CT Portal Venography in the Evaluation of Portosystemic Collateral Vessels in Liver Cirrhosis. RadioGraphics. 2002; 22: 1053-1061.

11. Zhao LQ, He W, Ji M, Liu P, Li P. 64-row multidetector computed tomography portal venography of gastric variceal collateral circulation. World J Gastroenterol , 2010; 16(8): 1003-1007

12. ELKammash T., ELFiky I., Zaiton F. Diagnostic performance of multidetector computed tomography in the evaluation of esophageal varices, The Egyptian Journal of Radiology and Nuclear Medicine, 2016; Volume 47, Issue 1.

13. Kim H, Choi D, Lee JH, Jo H, Gwak G, Koh C. High-risk esophageal varices in patients treated with locoregional therapy for HCC: assessment with liver computed tomography. World J Gastroenterol 2012;18(35):4905-11.

14.Antunez M., Gómez S, Merino-Casabiel X. Portosystemic shunts: A comprehensive review of the clinical and imaging features. Barcelona/ES 10.1594/ecr2014/C-1453.

15.Hegab AM and Luketic VA . Bleeding esophageal varices. How to treat this dreaded complication of portal hypertension. Postgraduate Medicine2001; 109 (2): 75- 86. 